Industrial Engineering

\& Management Systems

Vol 12, No 1, March 2013, pp.41-45

http://dx.doi.org/10.7232/iems.2013.12.1.041

ISSN 1598-7248 | EISSN 2234-6473 |

(C) 2013 KIIE

\title{
Some New Results on Uncertain Age Replacement Policy
}

\author{
Chunxiao Zhang*, Congrong Guo \\ College of Science, Civil Aviation University of China, Tianjin, China
}

(Received: September 3, 2012 / Revised: January 3, 2013 / Accepted: March 6, 2013)

\begin{abstract}
Age replacement policy is a commonly policy in maintenance management of spare part. It means that a spare part is always replaced at failure or fixed time after its installation, whichever occurs first. An optimal age replacement policy of spare parts concerns with finding the optimal replacement time determined by minimizing the expected cost per unit time. The age of the part was generally assumed to be a random variable in the past literatures, but in many situations, there are few or even no observed data to estimate the probability distribution of part's lifetime. In order to solve this phenomenon, a new uncertain age replacement policy has been proposed recently, in which the age of the part was assumed to be an uncertain variable. This paper discusses the optimal age replacement policies by dealing with the parts' lifetimes as different distributed uncertain variables. Several results on the optimal age replacement time are provided when the lifetimes are described by the uncertain linear, zigzag and lognormal distributions.
\end{abstract}

Keywords: Age Replacement Policy, Uncertain Renewal Process, Optimal Replacement Time, Uncertainty Distribution

* Corresponding Author, E-mail: cxzhang@cauc.edu.cn

\section{INTRODUCTION}

Among all kinds of maintenance policies, our major concerns are preventive maintenance and corrective maintenance. When corrective maintenance is carried out, the accidental failure of parts in a still operating system such as a flying aircraft, a running computer, a machine of manufacturing product, etc., is costly and dangerous, and it even brings bad reputation to enterprises. Therefore, preventive maintenance is needed to effectively avoid many breakdowns of parts in advance. However, preventive maintenance will generate too much expenses when it is carried out too frequently. So the best solution is a combination of these two kinds of maintenance. As a type of the combination maintenance, age replacement policy has been researched for a long time.

Barlow and Hunter (1960) firstly defined an age replacement policy as one in which a part is replaced at predetermined time $T$ or at failure, whichever occurs first. They studied the basic replacement policies under the common assumption that a system after repair is "as good as new". However, this assumption is not always reasonable in practice. On account of this fact, Brown and Proschan (1983) discussed an imperfect replacement model. Extensively, Tilquin and Cleroux (1975) proposed periodic replacement policy with adjustment costs. After that, Lin (1988) introduced a geometrical process to study two kinds of replacement policy for a simple repairable system based on the working age $T$ and the number of failures $N$, respectively, and the explicit expressions of the average cost rate under these two kinds of policy are determined. Furthermore, Zhang (1994) considered a bivariate replacement policy $(T, N)$ under which a unit is replaced at the working age $T$ or at the time of the $N$ th failure, whichever occurs first.

In those studies, the ages of parts were considered as random variables, and the failure distribution function based on the probability theory is applicable only when the obtained probability is close enough to the real frequency. However, few or even no observed data is available in some situation. Therefore, we have to invite some experts to evaluate their belief degree. Since humans tend to overweight unlikely events (Kahneman and Tversky, 1979), the belief degree may have much larger deviation than the frequency, and the probability theory is no longer valid. In this case, we have a choice to use the uncertainty theory to deal with this type of human uncertainty. The uncertainty theory was founded by Liu (2007), and refined by Liu (2010) based on normality, duality, subadditivity, and product axioms. Nowadays 
the uncertainty theory has become a branch of axiomatic mathematics for modeling human uncertainty and has been applied to many aspects.

In this paper, by dealing with the parts' lifetimes as uncertain variables, the optimal time of age replacement policy will be discussed when the lifetimes follow different uncertain distributions. The rest of this paper is organized as follows: Section 2 recalls some basic concepts and properties about the uncertain theory which will be used throughout the paper. In Section 3, the optimal replacement time of the age replacement policy is discussed when the lifetimes of the parts follow uncertain linear, zigzag and lognormal distribution. Finally, concluding remarks are given in Section 4.

\section{PRELIMINARIES}

In this section, we will introduce useful definitions and theorems of uncertainty theory that are applied throughout this paper.

Let $\Gamma$ be a nonempty set. A collection $L$ of $E\left[1 / \xi_{1}\right]$ is called a $\sigma$-algebra (a) if $\Gamma \in L$;(b) if $\Lambda \in L$, then $\Lambda^{c} \in L$; and (c) if $\Lambda_{1}, \Lambda_{2}, \cdots \in L$, then $\Lambda_{1} \cup \Lambda_{2} \cup \cdots \in L$. Each element $\Lambda$ in the $\sigma$-algebra $L$ is called an event. Uncertain measure is a function from $L$ to $[0,1]$. In order to present an axiomatic definition of uncertain measure, it is necessary to assign to each event $\Lambda$ a number $M\{\Lambda\}$ which indicates the belief degree that the event $\Lambda$ will occur. In order to ensure that the number $M\{\Lambda\}$ has certain mathematical properties, Liu (2007) proposed the following three axioms:

Axiom 1 (Normality Axiom). $M\{\Gamma\}=1$ for the universal set $\Gamma$.

Axiom 2 (Duality Axiom). $M\{\Lambda\}+M\left\{\Lambda^{c}\right\}=1$ for any event $\Lambda$.

Axiom 3 (Subadditivity Axiom). For every countable sequence of events $\Lambda_{1}, \Lambda_{2}, \cdots$ we have

$$
M\left\{\bigcup_{i=1}^{\infty} \Lambda_{i}\right\} \leq \sum_{i=1}^{\infty} M\left\{\Lambda_{i}\right\}
$$

Besides, in order to provide the operational law, Liu (2013) defined the product uncertain measure on the product $\sigma$-algebre $L$ as follows,

Axiom 4 (Product Axiom). Let $\left(\Gamma_{k}, L_{k}, M_{k}\right)$ be uncertainty spaces for $k=1,2, \cdots$ Then the product uncertain measure $M$ is an uncertain measure satisfying

$$
M\left\{\prod_{i=1}^{\infty} \Lambda_{k}\right\}=\stackrel{\wedge}{\wedge=1}_{\wedge} M_{k}\left\{\Lambda_{k}\right\},
$$

where $\Lambda_{k}$ are arbitrarily chosen events from $L_{k}$ for $k=$ $1,2, \cdots$, respectively.

Definition 1 (Liu, 2007). The set function $M$ is called an uncertain measure if it satisfies the normality, duality, and subadditivity axioms.

Definition 2 (Liu, 2007). An uncertain variable is a measurable function $\xi$ from the uncertainty space $(\Gamma$, $L, M)$ to the set of real numbers, i.e., for any Borel set $B$ of real numbers, the set

$$
\{\xi \in B\}=\{\gamma \in \Gamma \mid \xi(\gamma) \in B\}
$$

is an event.

Definition 3 (Liu, 2007). The uncertainty distribution $\Phi$ of an uncertain variable $\xi$ is defined by

$$
\Phi(x)=M\{\xi \leq x\}
$$

for any real number $x$.

Definition 4 (Liu, 2009). The uncertain variables $\xi_{1}, \xi_{2}$, $\cdots, \xi_{m}$ are said to be independent if

$$
M\left\{\bigcap_{i=1}^{m}\left(\xi_{i} \in B_{i}\right)\right\}=\stackrel{m}{i=1}_{\wedge}^{m}\left\{\xi_{i} \in B_{i}\right\}
$$

for any Borel sets $B_{1}, B_{2}, \cdots, B_{m}$ of real numbers.

Theorem 1 (Liu, 2010). Let $\xi_{1}, \xi_{2}, \cdots, \xi_{n}$ be independent uncertain variables with continuous uncertainty distributions $\Phi_{1}, \Phi_{2}, \cdots, \Phi_{n}$, respectively. If the function $f\left(x_{1}, x_{2}, \cdots, x_{n}\right)$ is strictly increasing with respect to $x_{1}, x_{2}, \cdots, x_{m}$ and strictly decreasing with respect to $x_{m+1}, x_{m+2}, \cdots, x_{n}$, then

$$
\xi=f\left(\xi_{1}, \xi_{2}, \cdots, \xi_{n}\right)
$$

is an uncertain variable with uncertainty distribution

$$
\Psi(x)=\sup _{f\left(x_{1}, x_{2}, \cdots, x_{n}\right)=x}\left(\min _{1 \leq i \leq m} \Phi_{i}\left(x_{i}\right) \wedge \min _{m+1 \leq i \leq n}\left(1-\Phi_{i}\left(x_{i}\right)\right)\right) .
$$

Corollary 1 (Liu, 2013). Let $\xi_{1}, \xi_{2}, \cdots, \xi_{n}$ be iid uncertain variables.

Then $\xi_{1}+\xi_{2}+\cdots+\xi_{n}$ and $n \xi$ are identically distributed uncertain variables. In other words, if $\Phi$ is the common uncertainty distribution of $\xi_{1}, \xi_{2}, \cdots, \xi_{n}$, then $\frac{1}{n} \sum_{i=1}^{n} \xi_{i}$ has also the uncertainty distribution $\Phi$ (but not independent of $\xi_{1}, \xi_{2}, \cdots, \xi_{n}$ ).

Expected value is the average of an uncertain variable in the sense of uncertain measure. It is an important index to rank uncertain variables.

Definition 5 (Liu, 2007). Let $\xi$ be an uncertain variable. Then the expected value of $\xi$ is defined by 


$$
E[\xi]=\int_{0}^{+\infty} M\{\xi \geq r\} d r-\int_{-\infty}^{0} M\{\xi \leq r\} d r
$$

provided that at least one of the two integrals is finite.

An uncertain process (Liu, 2008) is essentially a sequence of uncertain variables indexed by time. As an important uncertain process, a renewal process is an uncertain process in which events occur continuously and independently of one another in uncertain times.

Definition 6 (Liu, 2008). Let $\xi_{1}, \xi_{2}, \cdots$ be iid positive uncertain variables. Define $S_{0}=0$ and $S_{n}=\xi_{1}+\xi_{2}+\cdots$ $+\xi_{n}$ for $n \geq 1$. Then the uncertain process

$$
N_{t}=\max _{n \geq 0}\left\{n \mid S_{n} \leq t\right\}
$$

is called an uncertain renewal process.

For the uncertain renewal process, Liu (2010) proved that $N_{t} / t$ converges in distribution to $1 / \xi_{1}$ which is an uncertain variable. Based on this, Liu (2010) proved the elementary renewal theorem, i.e.,

$$
\lim _{t \rightarrow \infty} E\left[\frac{N_{t}}{t}\right]=E\left[\frac{1}{\xi_{1}}\right]
$$

under the assumption that $E\left[1 / \xi_{1}\right]$ exists.

\section{NEW THEOREMS ON UNCERTAIN AGE REPLACEMENT POLICY}

Age replacement policy in uncertain environment has been proposed by Yao and Ralescu (2013). Age replacement means that a part is always replaced at failure or at time $T$. Assume that the lifetimes of the parts are iid uncertain variables $\xi_{1}, \xi_{2}, \cdots$ with a common uncertainty distribution $\Phi$. Then the actual lifetimes of the parts are iid uncertain variables

$$
\xi_{1} \wedge T, \xi_{2} \wedge T, \cdots
$$

which may generate an uncertain renewal process

$$
N_{t}=\max _{n \geq 0}\left\{n \mid \sum_{i=1}^{n}\left(\xi_{i} \wedge T\right) \leq t\right\} .
$$

Let $B$ denote the "failure replacement" cost of replacing an element when it fails earlier than $T$, and $A$ the "planned replacement" cost of replacing an element at the age $T$. Define

$$
f(x)=\left\{\begin{array}{lll}
B, & \text { if } & x<T \\
A, & \text { if } & x=T .
\end{array}\right.
$$

Then $f\left(\xi_{i} \wedge T\right)$ is just the cost of replacing the $i$ th element, and the average replacement cost before the time $t$ is

$$
\frac{1}{t} \sum_{i=1}^{N_{t}} f\left(\xi_{i} \wedge T\right) .
$$

Yao and Ralescu (2013) proved that

$$
\lim _{t \rightarrow \infty} \frac{1}{t} \sum_{i=1}^{N_{t}} f\left(\xi_{i} \wedge T\right)=\frac{f\left(\xi_{1} \wedge T\right)}{\xi_{1} \wedge T}
$$

in the sense of convergence in distribution.

Theorem 2 (Yao and Ralescu, 2013). Let $\xi_{1}, \xi_{2}, \cdots$ be a sequence of iid positive uncertain variables with a common uncertainty distribution $\Phi$, and $N_{t}$ be an uncertain renewal process with uncertain interarrivals $\xi_{1} \wedge T, \xi_{2} \wedge T, \cdots$ Assume that

$$
f(x)=\left\{\begin{array}{lll}
B, & \text { if } & x<T \\
A, & \text { if } & x=T
\end{array}\right.
$$

with $0<A<B$. Then

$$
\lim _{t \rightarrow \infty} E\left[\sum_{I=1}^{N_{t}} f\left(\xi_{i} \wedge T\right) / t\right]=\frac{A}{T}+\frac{B-A}{T} \Phi(T)+B \int_{0}^{T} \frac{\Phi(x)}{x^{2}} d x .
$$

In order to find the optimal replacement time, we just need to find $T^{*}$ which solves the problem

$$
\min _{T} C(T)=\frac{A}{T}+\frac{B-A}{T} \Phi(T)+B \int_{0}^{T} \frac{\Phi(x)}{x^{2}} d x .
$$

Next, we discuss the optimal age replacement policy when the lifetime of unit follows different uncertainty distribution.

Theorem 3. Let $\xi_{1}, \xi_{2}, \cdots$ be a sequence of iid positive uncertain variables with a common linear uncertainty distribution $L(a, b)(a>0)$, and $N_{t}$ be an uncertain renewal process with uncertain interarrivals $\xi_{1} \wedge T, \xi_{2} \wedge$ $T, \cdots$. Assume that

$$
f(x)=\left\{\begin{array}{lll}
B, & \text { if } & x<T \\
A, & \text { if } & x=T .
\end{array}\right.
$$

with $0<A<B$. Then the optimal age replacement time is

$$
T^{*}=\frac{A b}{B} .
$$

And the minimum average age replacement cost is 


$$
C\left(T^{*}\right)=\frac{B}{b-a}\left(1+\ln \frac{A b}{B a}\right)-\frac{A}{b-a} .
$$

Proof. The linear uncertainty distribution $L(a, b)$ (Liu, 2013) is

$$
\Phi(x)=\left\{\begin{array}{clc}
0, & \text { if } \quad x \leq a \\
(x-a) /(b-a), & \text { if } \quad a \leq x \leq b \\
1, & \text { if } \quad x \geq b .
\end{array}\right.
$$

Then

$$
C(T)=\left\{\begin{array}{cl}
A / T, & \text { if } \quad T \leq a \\
\frac{A b}{b-a} \frac{1}{T}+\frac{B}{b-a} \ln T-\frac{A}{b-a}-\frac{B}{b-a} \ln a, & \text { if } \quad a \leq T \leq b \\
\frac{B}{b-a} \ln \frac{b}{a}, & \text { if } \quad T \geq b .
\end{array}\right.
$$

We can find the optimal age replacement time and the minimum cost in three intervals

$$
\begin{aligned}
& T^{*}=\left\{\begin{array}{clc}
a, & \text { if } & T \leq a \\
\frac{A b}{B}, & \text { if } & a \leq T \leq b \\
b, & \text { if } & T \geq b .
\end{array}\right. \\
& C\left(T^{*}\right)=\left\{\begin{array}{cl}
A / a, & \text { if } \quad T \leq a \\
\frac{B}{b-a}\left(1+\ln \frac{A b}{B a}\right)-\frac{A}{b-a}, & \text { if } \quad a \leq T \leq b \\
\frac{B}{b-a} \ln \frac{b}{a}, & \text { if } \quad T \geq b .
\end{array}\right.
\end{aligned}
$$

It is easy to verify that the expected cost of the second interval is the minimum cost. Therefore, the optimal replacement time is

$$
T^{*}=\frac{A b}{B} .
$$

The theorem is verified.

Theorem 4. Let $\xi_{1}, \xi_{2}, \cdots$ be a sequence of iid positive uncertain variables with a common zigzag uncertainty distribution $Z(a, b, c)(a>0)$, and $N_{t}$ be an uncertain renewal process with uncertain interarrivals $\xi_{1} \wedge T, \xi_{2} \wedge T, \cdots$ Assume that

$$
f(x)=\left\{\begin{array}{lll}
B, & \text { if } & x<T \\
A, & \text { if } & x=T .
\end{array}\right.
$$

with $0<A<B$. Then the optimal age replacement times is

$$
T^{*}=\left\{\begin{array}{cl}
\frac{A(2 b-a)}{B}, & \text { if } \quad 2 b-a \leq c \\
\frac{A c}{B}, & \text { if } 2 b-a \geq c .
\end{array}\right.
$$

And the minimum average age replacement cost is

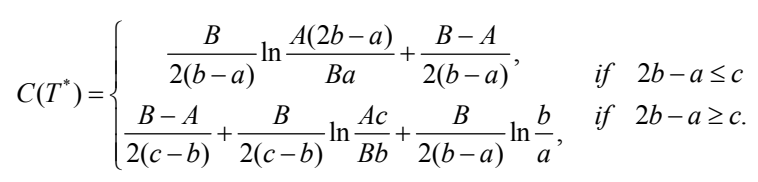

Proof. The uncertainty distribution of zigzag uncertain variable $Z(a, b, c)(\mathrm{Liu}, 2013)$ is

$$
\Phi(x)=\left\{\begin{array}{clc}
0, & \text { if } \quad x \leq a \\
(x-a) / 2(b-a), & \text { if } \quad a \leq x \leq b \\
(x+c-2 b) / 2(c-b), & \text { if } \quad b \leq x \leq c \\
1, & \text { if } \quad x \geq c .
\end{array}\right.
$$

Then

$$
C(T)= \begin{cases}A / T, & \text { if } T \leq a \\ \frac{2 b-a}{2(b-a)} \frac{A}{T}+\frac{B}{2(b-a)} \ln T-\frac{A}{2(b-a)}-\frac{B}{2(b-a)} \ln a, & \text { if } a \leq T \leq b \\ \frac{c}{2(c-b)} \frac{A}{T}+\frac{B}{2(c-b)} \ln T+\frac{B}{2(b-a)} \ln \frac{b}{a}-\frac{A}{2(c-b)}-\frac{B}{2(c-b)} \ln b, & \text { if } b \leq T \leq c \\ \frac{B}{2(b-a)} \ln \frac{b}{a}+\frac{B}{2(c-b)} \ln \frac{c}{b}, & \text { if } T \geq c,\end{cases}
$$

where the expected cost of the fourth interval is a constant. We can find the optimal age replacement time and the minimum cost in each interval

$$
\begin{gathered}
T^{*}=\left\{\begin{array}{cl}
a, & \text { if } T \leq a \\
\frac{A(2 b-a)}{B}, & \text { if } a \leq T \leq b \\
\frac{A c}{B}, & \text { if } \quad b \leq T \leq c
\end{array}\right. \\
C, \quad \text { if } T \geq c \\
C\left(T^{*}\right)=\left\{\begin{array}{cl}
A / a, & \text { if } T \leq a \\
\frac{B}{2(b-a)} \ln \frac{A(2 b-a)}{B a}+\frac{B-A}{2(b-a)}, & \text { if } a \leq T \leq b \\
\frac{B-A}{2(c-b)}+\frac{B}{2(c-b)} \ln \frac{A c}{B b}+\frac{B}{2(b-a)} \ln \frac{b}{a}, & \text { if } b \leq T \leq c \\
\frac{B}{2(b-a)} \ln \frac{b}{a}+\frac{B}{2(c-b)} \ln \frac{c}{b}, & \text { if } T \geq c .
\end{array}\right.
\end{gathered}
$$

It is easy to verify that the expected cost of the second or the third interval is the minimum cost. Therefore, the optimal replacement time is 


$$
T^{*}=\left\{\begin{array}{cl}
\frac{A(2 b-a)}{B}, & \text { if } \quad 2 b-a \leq c \\
\frac{A c}{B}, & \text { if } 2 b-a \geq c .
\end{array}\right.
$$

The theorem is verified.

Theorem 5. Let $\xi_{1}, \xi_{2}, \cdots$ be a sequence of iid positive uncertain variables that have a common lognormal uncertainty distribution $N(e, \sigma)$ with $\sigma<\pi / \sqrt{3}$, and $N_{t}$ be an uncertain renewal process with uncertain interarrivals $\xi_{1} \wedge T, \xi_{2} \wedge T, \cdots$ Assume that

$$
f(x)=\left\{\begin{array}{lll}
B, & \text { if } & x<T \\
A, & \text { if } & x=T .
\end{array}\right.
$$

with $0<A<B$. Then the optimal replacement time is

$$
T^{*}=\exp \left(e-\frac{\sqrt{3} \sigma}{\pi} \ln \left(\frac{(B-A) \pi}{A \sqrt{3} \sigma}-1\right)\right)
$$

under the condition described in Theorem 2.

Proof. The lognormal uncertainty distribution (Liu, 2013) is

$$
\begin{aligned}
& \Phi(x)=\left(1+\exp \left(\frac{\pi(e-\ln x)}{\sqrt{3} \sigma}\right)\right)^{-1}, \\
& \Phi^{\prime}(x)=\frac{\exp \left(\frac{\pi(e-\ln x)}{\sqrt{3} \sigma}\right)}{\left(1+\exp \left(\frac{\pi(e-\ln x)}{\sqrt{3} \sigma}\right)\right)^{2}} \cdot \frac{\pi}{\sqrt{3} \sigma} \cdot \frac{1}{T} .
\end{aligned}
$$

Therefore,

$$
\begin{aligned}
C^{\prime}(T) & =\frac{1}{T^{2}}\left[(B-A) T \Phi^{\prime}(T)-A+A \Phi(T)\right] \\
& =\frac{1}{T^{2}} \frac{\exp \left(\frac{\pi(e-\ln x)}{\sqrt{3} \sigma}\right)}{\left(1+\exp \left(\frac{\pi(e-\ln x)}{\sqrt{3} \sigma}\right)\right)^{2}} \\
& \times\left[(B-A) \frac{\pi}{\sqrt{3} \sigma}-A\left(1+\exp \left(\frac{\pi(e-\ln x)}{\sqrt{3} \sigma}\right)\right)\right] .
\end{aligned}
$$

Let $C^{\prime}(T)=0$, we can get the unique root

$$
T=\exp \left(e-\frac{\sqrt{3} \sigma}{\pi} \ln \left(\frac{(B-A) \pi}{A \sqrt{3} \sigma}-1\right)\right) .
$$

Obviously, $T$ is the optimal replacement time $T^{*}$. The theorem is verified.

\section{CONCLUSION}

In this paper, we discussed an optimal age replacement policy when the age of the unit follows different uncertain distributions, and the optimal time was given to minimize the long-run average replacing cost. In addition, three theorems were developed on the analytically optimal replacement policy under the assumption that the uncertain lifetimes follow linear distribution, zigzag distribution and lognormal distribution.

\section{ACKNOWLEDGMENTS}

This work is supported by the Fundamental Research Funds for the Central Universities under grant no. ZXH2012K005. The research is also supported by Uncertainty Theory Laboratory of Department of Mathematical Sciences, Tsinghua University, Beijing, China.

\section{REFERENCES}

Barlow, R. E. and Hunter, L. C. (1960), Optimum preventive maintenance policy, Operation Research, 8(1), 90-100.

Brown, M. and Proschan, F. (1983), Imperfect repair, Journal of Applied Probability, 20(4), 851-859.

Kahneman, D. and Tversky, A. (1979), Prospect theory: an analysis of decision under risk, Econometrica, 47(2), 263-292.

Lin, Y. (1988), Geometric processes and replacement problem, Acta Mathematicae Applicatae Sinica, 4 (4), 366-377.

Liu, B. (2007), Uncertainty Theory (2nd ed.), SpringerVerlag, Berlin.

Liu, B. (2008), Fuzzy process, hybrid process and uncertain process, Journal of Uncertain Systems, 2(1), 3-16.

Liu, B. (2009), Some research problems in uncertainty theory, Journal of Uncertain Systems, 3(1), 3-10.

Liu, B. (2010), Uncertainty Theory: A Branch of Mathematics for Modeling Human Uncertainty, SpringerVerlag, Berlin.

Liu, B. (2013), Uncertainty Theory (4th ed.), Uncertainty Theory Laboratory, Beijing.

Tilquin, C. and Cleroux, R. (1975), Periodic replacement with minimal repair at failure and adjustment costs, Naval Research Logistics Quarterly, 22(2), 243-254.

Yao, K. and Ralescu, D. A. (2013), Age replacement policy in uncertain environment, Iranian Journal of Fuzzy Systems, 10(2), 29-39.

Zhang, Y. L. (1994), A bivariate optimal replacement policy for a repairable system, Journal of Applied Probability, 31(4), 1123-1127. 